\title{
A Survey of Context-Aware Healthcare Recommender Systems
}

\author{
Mohamed Hussein Abdi ${ }^{1}$, George Onyango Okeyo ${ }^{2}$, Ronald Waweru Mwangi ${ }^{3}$ \\ ${ }^{1}$ Department of Information and Communication Technology, Kenya Medical Research Institute \\ ${ }^{2}$ Department of Computing, School of Computing \& Information Technology, Jomo Kenyatta University of Agriculture \& Technology \\ ${ }^{3}$ Department of Computing, School of Computing \& Information Technology, Jomo Kenyatta University of Agriculture \& Technology
}

\begin{abstract}
The advent of social web has transformed users from sheer consumers to ardent producers of information. The rapid growth coupled with the reliance on the internet for information ranging from e-commerce, e-government and social networks has led to infobesity. Recommender Systems have become essential tool to aid users to overcome the problem of information overload and provide personalized relevant suggestions. The use of Recommender Systems have been successful in both industry and academia. Recommender systems have also found their way into the Healthcare domain with varied applications.Despite the growing practical demand, a few challenges are hindering the adoption ofRecommender Systems in the Healthcare.The purpose of this study is to understand the trend of Recommender Systems applications inHealthcareby examining the published literature, and to provide practitioners and researchers with insight and future direction. We conducted a focused literature review to identify research papers on Recommender Systems in the Healthcare in the last ten years. The key findings of this survey is that the application of Recommender Systems inHealthcare is evolving. The incorporation of contextual information is limited although it has been suggested as a key ingredient to improving the quality of the recommendations and the accuracy of the predications.It is important to point out that aside from the common filtering problems of sparsity, cold start and scalability, a socio-technical issue of privacy, security and trust is emerging.Collaborative Filtering technique is predominantly in use in Healthcare Recommender Systems. Incorporation of contextual information is limited.Privacy, security and trust concerns may hinder the widespread use and acceptance of the Healthcare Recommender Systems.
\end{abstract}

Keywords: Collaborative Filtering,Context-Aware, HealthcareRecommender Systems, Quality Recommendations, Accurate Predictions, Privacy

\section{Introduction}

The primary purpose of personalized recommendation service is to provide items, products or information tailored to individuals, satisfying their needs in a given context, based on knowledge of their preferences and behavior [1]. Recommender Systems have been touted as beneficial to service providers and users [2]. The main aim of ecommerce sites is to increase revenues, customer satisfaction and retention. For users, Recommender Systems lower the transaction costs of finding and selecting items, and generally improve decision quality [3], [4].

HealthcareRecommender Systems (HRS) is a specialization of a Recommender Systems as defined in[5]. In the context of a Healthcare recommender system, a recommendable item of interest is a piece of non-confidential, scientifically proven or at least generally accepted medical information, which in itself is not linked to an individual's medical history [6].

Recommender Systems make recommendations using three fundamental steps: preferences acquisition from the user's input data, recommendation computation and recommendation presentation. Robin Burke [7]classified existing Recommender Systems into four fundamental categories namely content-based, collaborative, Knowledgebased and hybrid based filtering systems.
The different approaches used in providing recommendations suffer from various short comings namely data sparsity, scalability, cold-start and privacy concerns [1], [8]. Research in the past decade has been focused to addressing these limitations arising from the various approaches and techniques in order to improve the quality recommendations and accuracy of the predictions.

In order to solve some problems of traditional recommender system, Context-Aware Recommender System (CARS) has attracted the attention of the academic[9]. In Recommender Systems context is usually playing the role of additional information besides users, items and the ratings which may be relevant at the current time to make a recommendation. Context-Aware recommender systems aim to generate more relevant suggestions by adapting them to the user's contextual situation. Context-Aware systems sense and adapt their behavior based on the changing contexts [10], which generally consists of four basic components: Context Acquisition, Context Discovery, Context Model, and Context Processing [11].

The aim of study is to understand the trend of recommender systems applications in Healthcare domain by examining the published literature, and to provide practitioners and researchers with insight and future direction on recommender systems in this area.The rest of this paper is organized as follows:In section 2 we describe our methodology in this research and present an overview of HealthcareRecommender Systems in section 3. This will be 


\section{International Journal of Science and Research (IJSR) \\ ISSN (Online): 2319-7064}

Index Copernicus Value (2016): 79.57 | Impact Factor (2015): 6.391

followed by discussions and conclusions in subsequent sections.

\section{Methodology / Approach}

An effective literature review should involve the leading literature as it is likely to cover the major contributions [12]. Accordingly, we searched in all Quality Information Systems Literature stated in [13] that were accessible from our academic environment and also from our digital library available through professional membership to Association of Computing Machinery (ACM) and IEEE computer Society. In order not to miss any relevant documents, we preferred to perform a broad research and eliminate the irrelevant documents manually.

The Identification of relevant literature (Fig. 1) involved search through the following electronic resources: The ACM
Digital Library, IEEE Computer Society, JKUAT Resources and Google Scholar. We used the keywords of "Recommender Systems", "Health Recommender System" and "Healthcare Recommender System". Each time, we repeated the search also with the keyword "Health or Healthcare" to cover different writing styles and areas of interest. The initial refinement was made considering two criteria: publications within the last ten years $(2006-2016)$ and evaluating the quality of the journals using impact factor and citation rates. In total 61 papers were retrieved. Removing duplicates and refining by title basis reduced the number to 52 and to 25 papers by abstract-basis elimination. In the next phase, 14 papers were found meeting the quality criteria: reliability of the source, integrity in the content and providing applicable studies. In the final phase, findings were synthesized and reported.

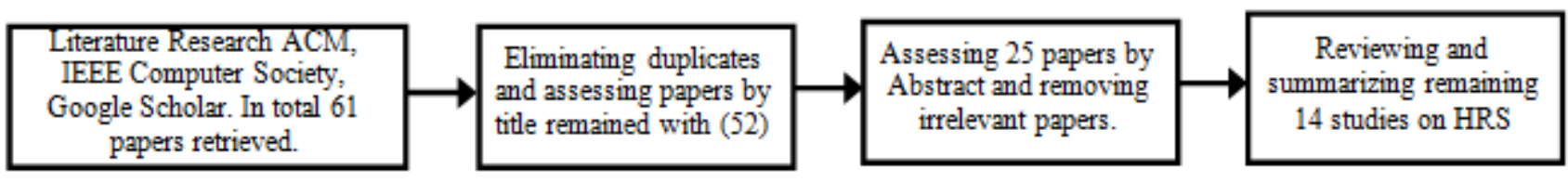

Figure 1: Review methodology

The search was implemented on all sources that were accessible through these electronic databases: Journals, conference proceedings, books, reference works, online reports and magazine articles.

\section{Literature survey on Healthcare Recommender Systems (HRS)}

The adoption of Information and Communication Technology in the Healthcare sector led to the concept of eHealth, computer based systems that stored information regarding diseases, diagnoses and different treatments. To provide personalized recommendation to patients, a recommender system must access the information stored in Personal Health Record Systems. It is critical to preserve the privacy of patients so as to maintain the user satisfaction and trust in the offered services.

Fernandez-Luque et al. [14]highlighted the application of recommender system in Healthcare to provide personalized health education. The study suggested the use of ComputerTailoring Health Education Systems (CTHES) and social networking to deliver content and also to harvest knowledge about the users and the content. They provided a comparison between CTHES and Recommender Systems applications due to lack of previous studies in the two fields and outlined the necessity for adoption of Recommender Systems to overcome the challenge of health information overload.

The limitations of Computer Tailored Health Communication (CTHC) systems and the advantages of incorporating Recommender Systems into CTHC and the challenges thereto were presented in [15]. They argue that CTHC has reached its natural limits although technological advances have enabled CTHC to be delivered on multiple platforms like websites, email, and mobile devices and to reach large populations.
There have been approaches to make recommendations considering the data stored in Electronic Health Record. López-Nores et al. presented a number of enhancements that enable Digital Television (DTV) Recommender Systems to exploit the synergies between healthcare and areas like entertainment, electronic commerce, and education[16]. The study raised privacy concerns regarding the storing of sensitive patients data in a remote sever noting that nothing stops server owners from exploiting this sensitive information. The main emphasis is on the privacy of the users and only recommends items delivered to all the TV viewers through the broadcast networks. While this limitation is acknowledged the lack of context of the TV viewers affects the quality of the recommendation and the accuracy of the prediction.

The work in [17] present research on integrating personal health data in Personal Health Record (PHR) into Recommender Systems. The approach exploits the existing semantic networks like Wikipedia to obtain a health graph data structure representing health related concepts which can be used to compute the semantic distances among the pairs of such concepts. A ranking procedure based on the health graph enables a match between entries of a PHR system and health information artifacts. The generated Health graphs are language specific.

A Health social network is an online information service which facilitates information sharing between closely related members of a community. Thus, a health social network provides emotional support by allowing patients to find others in similar health situations. This type of social network has shown great potential to empower patient selfcare. PatientsLikeMe and GenNet are examples of patientdriven Healthcare Social Network Sites (SNS) that encourages information exchange and collaboration between patients and between patients and doctors. GenNet Health- 


\section{International Journal of Science and Research (IJSR) \\ ISSN (Online): 2319-7064}

Index Copernicus Value (2016): 79.57 | Impact Factor (2015): 6.391

Care Social Network, a collaborative virtual environment that aims health promotion to patients with physical disorders caused by genetic diseases [18].

The recommendation based on social behaviors of users in online health communities was proposed by [19] to address the problem of recommendation using multiple relations with wide-ranging implicit ratings that are derived from social behavior.

A recommender system for constructing nursing care plans for elderly people using correlations among nursing diagnoses, outcome and interventions [20]to provide clinical decision support, nursing education and clinical quality control. The study clearly acknowledges and suggests the need for incorporating contextual information into the recommendation process.

Reminiscence Therapy Enhanced Material Profiling in Alzheimers and other Dementias (REMPAD) system [21] profiles people with Alzheimer's and other dementias, and provides multimedia content tailored to a given group context. The recommendation approach uses group member preferences using case-based reasoning (CBR) combined with a content- based method recommending multimedia content for use in group reminiscence therapy for people with Alzheimer's and other dementias. While the approach suffers the limitations of the case-based reasoning and content-based approach and it further lacks the use of contextual information in group recommendations.

Other studies include predicting mental health from web usage behavior [22], collaborative filtering-based Computer Tailored Health Communication(CTHC) to provide messages tailored to individual patients [23], Health recommendation system to provide personalized nutrition advice [24]. Patient Experience Recommender System for Persuasive Communication Tailoring (PERSPeCT) was proposed, a system developed for use in a clinical trial of CTHC-based smoking cessation support interventions was proposed by [25]. The collaborative filtering based recommender system was to address the problem static, expert written rules in CTHC systems.

\section{Context-Aware Healthcare Recommender Systems}

The traditional two-dimensional Recommender Systems provide recommendations of items to users and vice versa without taking into consideration the circumstances and other contextual information recommendation take place. In order to solve some problems of traditional recommender system, Context-Aware Recommender System (CARS) has attracted the attention of the academic [9]. In context plays the role of additional information besides users, items and the ratings which may be relevant at the current time to make a recommendation.

Context-Aware Recommender system for the Elderly (CARE) presents a study that combines the functionality of a digital image frame with an active recommender mode [26] using data derived from personal user and incorporating contextual data collected with sensors integrated into the users' mobile phone. The recommendations are chosen on the basis of context information acquired by sensors embedded in the user's environment, and a well-being model to carefully decide on at which point in time what kind of activity is most suitable to suggest. The contextual information in this study is limited to time ((morning, forenoon, lunchtime, afternoon, evening, night), ) and weather conditions (very Good, Good, Fair, Bad, very Bad). Activity-awareness for human-engaged wellness applications (ATHENA) and Knowledge Acquisition and Reasoning Engine (KARE) [27] is a platform designed to integrate relationship between the basic health primitives of the users and estimates their lifestyle to generate real-time recommendations for their wellbeing. Specifically the platform engine (KARE) recommends food, mental and physical therapy to the ATHENA users that are based on their personal preferences, historical physical, mental and social health information. While the study mentions Context-Aware recognizer, the contextual information is not explicitly mentioned.

Other related works includes a disease prediction model extending the collaborative filtering technique used in Recommender Systems was developed [28] using publicly available patients' social network data to predict correlation relationships in diseases and Health-aware Food Recommender System [29]. A semantic recommender system able to suggest doctors and hospitals that best fit a specific patient profile [30]. Others include aRecommender System for Medical Imaging Diagnostic[31]and Friend recommendation for healthy weight in social networks[32].

Table 1 provides a summary of the health Recommender Systems and their recommendation techniques. Although the need for context-based, individually tailored health information in personal health records is expressed [6], [26], [33], Context-Aware Healthcare Recommender Systems has received little attention in health Recommender Systems literature.

Table 1: Summary of HRSbased recommendation techniques

\begin{tabular}{|c|c|c|c|c|}
\hline No. & Recommender Systems & Collaborative & $\begin{array}{l}\text { Context- } \\
\text { Aware }\end{array}$ & Hybrid \\
\hline 1 & $\begin{array}{l}\text { Healthcare information systems: data mining methods in the creation of a clinical } \\
\text { recommender system [20] }\end{array}$ & $\checkmark$ & - & - \\
\hline 2 & Context-Aware based Item Recommendation for Personalized Service [34] & $\checkmark$ & $\checkmark$ & - \\
\hline 3 & Predicting Mental Health Status in the Context of Web Browsing [22] & $\checkmark$ & - & - \\
\hline 4 & Recommendation in online health communities [19] & $\checkmark$ & - & - \\
\hline 5 & $\begin{array}{l}\text { Towards Collaborative Filtering Recommender Systems for Tailored Health } \\
\text { Communications [23] }\end{array}$ & $\checkmark$ & - & - \\
\hline 6 & GenNet Health-Care Social Network [18] & $\checkmark$ & - & - \\
\hline
\end{tabular}

Volume 6 Issue 12, December 2017 
International Journal of Science and Research (IJSR)

ISSN (Online): 2319-7064

Index Copernicus Value (2016): 79.57 | Impact Factor (2015): 6.391

\begin{tabular}{|c|c|c|c|c|}
\hline No. & Recommender Systems & Collaborative & $\begin{array}{l}\text { Context- } \\
\text { Aware }\end{array}$ & Hybrid \\
\hline 7 & $\begin{array}{l}\text { Patient Experience Recommender System for Persuasive Communication Tailoring } \\
\text { (PERSPeCT) [25] }\end{array}$ & $\checkmark$ & - & - \\
\hline 8 & Context-Aware Recommender system for the Elderly (CARE) [26] & $\checkmark$ & $\checkmark$ & $\checkmark$ \\
\hline 9 & Activity-awareness for human-engaged wellness applications (ATHENA) [27] & $\checkmark$ & - & - \\
\hline 10 & A Recommender System for Medical Imaging Diagnostic [31] & $\checkmark$ & - & - \\
\hline 11 & Context-Aware Recommender for Smart Health [35] & $\checkmark$ & $\checkmark$ & - \\
\hline 12 & $\begin{array}{l}\text { DIETOS: a recommender system for adaptive diet monitoring and personalized food } \\
\text { suggestion[36] }\end{array}$ & $\checkmark$ & - & $\checkmark$ \\
\hline 13 & Application of Recommender System Methods for Therapy Decision Support[37] & $\checkmark$ & - & $\checkmark$ \\
\hline 14 & $\begin{array}{c}\text { A Fog Based Middleware for Automated Compliance With OECD Privacy Principles in } \\
\text { Internet of Healthcare Things [38] }\end{array}$ & $\checkmark$ & - & - \\
\hline
\end{tabular}

There are many solutions that have been proposed to address the privacy concerns in Recommender Systems in general but limited on the healthcare. Prior research on preserving recommendation systems has three main focuses: Security, privacy and trust.

A reliable doctor Recommender Systems [39] propose a Secure Processing Architecture (SPA) and Anonymous Contributions Architecture (ACA) in order to protect the privacy of sensitive patient health records, provide reliable information and meet the functional requirements of a Recommender System. The proposed framework enables patients to gather reliable doctor recommendations for their condition(s) while protecting the privacy of patients' contribution their ratings and the patients making inquiries. To enhance the reliability of the recommendations, the framework is said to be capable of protecting the users from dishonest users and physicians.

In [38] a middleware called personal gateway is proposed for cloud based Internet of Healthcare things that exposes sufficient amount of information while at the same time guarding against unauthorized access to users personal Health data.

\section{Results and Discussion}

There is increasing dependence on the internet for health related information on the internet. To address this demand, there is also an increase in the number of social network sites that provide healthcare information leading to information overload. The CTHC has been in existence for many years to address communications needs in Healthcare. Despite technological advances, CTHC is no longer able to meet the personalization and relevance requirements. One notable weakness of many existing CTHC systems is that they are based on expert-written rules and thus have no ability to learn from their users over time. The application of Recommender Systems in Healthcare has greatly overcome this problem.However, privacy, security and trust concerns are more pronounced in the healthcare due to the DoctorPatient confidentiality requirements. The Recommender Systems in Healthcare must therefore meet both the functional, privacy, security and trust requirements. Patientdriven healthcare social network sites are gaining popularity as a forum to share information on personal Health related issues. All the existing recommendation approaches can be used to provide healthcare recommendations. However, collaborative filtering has emerged as a popular approach.
However, the approaches lack the incorporation of contextual information.

\section{Conclusion and Future Work}

In this paper we have contributed a comprehensive literature in the field of HealthcareRecommender Systems. We provided background in the field Recommender Systems, motivating the work and outlining the limitations of existing approaches. We have also outlined the existing recommendation approaches in healthcare Recommender Systems. We conclude that the application of Recommender Systems in healthcare is varied and gradually evolving. The incorporation of contextual information is limited althoughit has been acknowledged as crucial to the recommendation process.

Based on the evidence presented, we propose a future research agenda of developing a taxonomy of and the incorporation of contextual information in healthcareRecommender Systems. It is also evident that privacy, security and trust concerns that is inherent in the traditional Recommender Systems needs to be addressed as well.

\section{References}

[1] G. Adomavicius and A. Tuzhilin, "Toward the next generation of recommender systems: A survey of the state-of-the-art and possible extensions," IEEE Trans. Knowl. Data Eng., vol. 17, no. 6, pp. 734-749, 2005.

[2] P. Cremonesi, F. Garzotto, and R. Turrin, "Investigating the Persuasion Potential of Recommender Systems from a Quality Perspective," ACM Trans. Interact. Intell. Syst., vol. 2, no. 2, pp. 141, 2012.

[3] A. Azaria, A. Hassidim, and S. Kraus, "Movie recommender system for profit maximization," Proc. 7th ACM Conf. Recomm. Syst. - RecSys '13, pp. 121128, 2013.

[4] A. J. P. Jeckmans, M. Beye, Z. Erkin, P. Hartel, R. L. Lagendijk, and Q. Tang, "Privacy in Recommender Systems," Soc. Media Retr., pp. 263-281, 2013.

[5] G. Adomavicius and A. Tuzhilin, "Context-Aware Recommender Systems," in Recommender Systems Handbook, F. Ricci, L. Rokach, B. Shapira, and P. B. Kantor, Eds. Springer, US, 2011, pp. 217-250.

[6] M. Wiesner and D. Pfeifer, "Health recommender systems: Concepts, requirements, technical basics and 


\section{International Journal of Science and Research (IJSR) \\ ISSN (Online): 2319-7064}

Index Copernicus Value (2016): 79.57 | Impact Factor (2015): 6.391

challenges," Int. J. Environ. Res. Public Health, vol. 11, no. 3, pp. 2580-2607, 2014.

[7] R. Burke, "Hybrid Recommender Systems: Survey and experiments," User Model. UserAdapted Interact., vol. 12, no. 4, pp. 331-370, 2002.

[8] H. H. Moe and W. T. Aung, "Building Ontologies for Cross-domain Recommendation on Facial Skin Problem and Related Cosmetics," Int. J. Inf. Technol. Comput. Sci., vol. 6, no. 6, pp. 33-39, 2014.

[9] H. Liu, H. Zhang, K. Hui, H. He, and C. Science, "Overview Of Context-aware Recommender System Research," 3rd Int. Conf. Mechatronics, Robot. Autom., no. Icmra, pp. 1218-1221, 2015.

[10] J. Coutaz, J. L. Crowley, S. Dobson, and D. Garlan, "Context is key," Commun. ACM, vol. 48, no. 3, p. 49, 2005.

[11] M. Baldauf, S. Dustdar, and F. Rosenberg, "A survey on context-aware systems," Int. J. Ad Hoc Ubiquitous Comput., vol. 2, no. 4, pp. 263-277, 2007.

[12] J. Webster and R. T. Watson, "Analyzing the Past to Prepare for the Future: Writing a Literature Review.," $M I S Q$., vol. 26, no. 2, pp. xiii-xxiii, 2002.

[13] Y. Levy and T. J. Ellis, "A Systems Approach to Conduct an Effective Literature Review in Support of Information Systems Research," Inf. Sci. J., vol. 9, no. 1, pp. 182-212, 2006.

[14] L. Fernandez-Luque, R. Karlsen, and L. K. Vognild, "Challenges and opportunities of using recommender systems for personalized health education," Stud. Health Technol. Inform., vol. 150, pp. 903-907, 2009.

[15] R. S. Sadasivam et al., "Collective-intelligence recommender systems: Advancing computer tailoring for health behavior change into the 21 st century," $J$. Med. Internet Res., vol. 18, no. 3, pp. 1-14, 2016.

[16] M. López-Nores, Y. Blanco-Fernändez, J. J. PazosArias, and J. García-Duque, "Exploring synergies between digital tv recommender systems and electronic health records," Proc. 8th Int. Interact. Conf. Interact. TV\&Video - EuroITV'10, p. 127, 2010.

[17] M. Wiesner and D. Pfeifer, "Adapting recommender systems to the requirements of personal health record systems," Proc. 1st ACM Int. Heal., pp. 410-414, 2010.

[18] C. V. C. De Magalhães, E. Souza, J. da S. C. Neto, and G. Vilar, "Recommender Systems: an Experience With GenNet Health-Care Social Network," in The Fifth International Conference on eHealth, Telemedicine, and Social Medicine Recommender, 2013, no. c, pp. 276-279.

[19] S. P. Crain, K. Zhou, and H. Zha, "Recommendation in Online Health Communities," Proc. 2012 IEEE/ACM Int. Conf. Adv. Soc. Networks Anal. Min. Recomm., 2012.

[20] L. Duan, W. N. Street, and E. Xu, "Healthcare information systems: data mining methods in the creation of a clinical recommender system," Enterp. Inf. Syst., vol. 5, no. 2, pp. 169-181, 2011.

[21] A. Bermingham, J. O’Rourke, C. Gurrin, R. Collins, K. Irving, and A. F. Smeaton, "Automatically recommending multimedia content for use in group reminiscence therapy," Proc. 1st ACM Int. Work. Multimed. Index. Inf. Retr. Healthc. - MIIRH '13, pp. 49-58, 2013.
[22] D. Nie, Y. Ning, and T. Zhu, "Predicting mental health status in the context of web browsing," in Proceedings of the 2012 IEEE/WIC/ACM International Conference on Web Intelligence and Intelligent Agent Technology Workshops, WI-IAT 2012, 2012, no. Im, pp. 185-189.

[23] B. M. Marlin, R. J. Adams, R. Sadasivam, and T. K. Houston, "Towards collaborative filtering recommender systems for tailored health communications.," AMIA Аnпu. Symp. Proc., vol. 2013, pp. 1600-7, 2013.

[24] S. Wendel, B. G. C. Dellaert, A. Ronteltap, and H. C. M. van Trijp, "Consumers' intention to use health recommendation systems to receive personalized nutrition advice.," BMC Health Serv. Res., vol. 13, no. 1, p. 126, 2013.

[25] R. J. Adams, R. S. Sadasivam, and K. Balakrishnan, "PERSPeCT: collaborative filtering for tailored health communications," in RecSys'14, 2014, pp. 329-332.

[26] T. Rist, A. Seiderer, S. Hammer, M. Mayr, and E. Andr, "CARE - Extending a Digital Picture Frame with a Recommender Mode to Enhance Well-Being of Elderly People," in the proceedings of the 2015 9th International Conference on Pervasive Computing Technologies for Healthcare (PervasiveHealth), 2015.

[27] R. Ali, M. H. Siddiqi, B. H. Kang, and S. Lee, "KARE: A Hybrid Reasoning Approach for Promoting Active Lifestyle," Proc. 9th Int. Conf. Ubiquitous Inf. Manag. Commun., p. 72:1--72:5, 2015.

[28] X. Ji, S. A. Chung, and J. Geller, "A Collaborative Filtering Approach to Assess Individual Condition Risk Based on Patients' Social Network Data," $B C B$ '14 Proc. 5th ACM Conf. Bioinformatics, Comput. Biol. Heal. Informatics Pages 639-640, pp. 639-640, 2014.

[29] M. Ge, F. Ricci, and D. Massimo, "Health-aware Food Recommender System," Proc. 9th ACM Conf. Recomm. Syst., pp. 333-334, 2015.

[30] F. Narducci, C. Musto, M. Polignano, M. De Gemmis, P. Lops, and G. Semeraro, "A Recommender System for Connecting Patients to the Right Doctors in the HealthNet Social Network," in WWW 2015 Companion, 2015, pp. 81-82.

[31] E. Monteiro, F. Valente, C. Costa, and J. L. Oliveira, "A Recommender System for Medical Imaging Diagnostic," Stud. Health Technol. Inform., vol. 210, pp. 461-463, 2015.

[32] A. Li, E. W. T. Ngai, and J. Chai, "Friend recommendation for healthy weight in social networks A novel approach to weight loss Anming," Ind. Manag. Data Syst., vol. 20, no. 3, pp. 237-248, 2015.

[33] Y. Zhang, "Contextualizing consumer health information searching: an analysis of questions in a social Q\&A community," Proc. 1st ACM Int. Heal., pp. 210-219, 2010.

[34] J. H. Kim, D. Lee, and K. Y. Chung, "Context-aware based item recommendation for personalized service," 2011 Int. Conf. Inf. Sci. Appl. ICISA 2011, 2011.

[35] F. Casino, E. Batista, C. Patsakis, and A. Solanas, "Context-aware recommender for smart health," 2015 IEEE 1st Int. Smart Cities Conf. ISC2 2015, 2015.

[36] G. Agapito et al., "DIETOS: A recommender system for adaptive diet monitoring and personalized food suggestion," Int. Conf. Wirel. Mob. Comput. Netw. 


\section{International Journal of Science and Research (IJSR) \\ ISSN (Online): 2319-7064}

Index Copernicus Value (2016): 79.57 | Impact Factor (2015): 6.391

Commun., 2016.

[37] F. Graßer et al., "Application of recommender system methods for therapy decision support," 2016 IEEE 18th Int. Conf. e-Health Networking, Appl. Serv. Heal. 2016, 2016.

[38] A. M. Elmisery, S. Rho, and D. Botvich, "A fog based middleware for automated compliance with OECD privacy principles in internet of healthcare things," IEEE Access, vol. 4, no. Idc, pp. 8418-8441, 2016.

[39] T. R. Hoens, B. Marina, S. Aaron, and V. C. Nitesh, "Reliable medical recommendation systems with patient privacy," ACM Trans. Intell. Syst. Technol., vol. 4, no. 4, pp. 1-31, 2013.

\section{Author Profile}

Mr. Mohamed H. Abdi, is a Ph.D Student in Information Technology at Jomo Kenyatta University of Agriculture \& Technology.

George O. Okeyo, is the Chairman of the Information \& Communication Technology and the main supervisor.

Ronald WaweruMwangi'is a Professor in the School of Computing \& Information Technology and the second supervisor.

Volume 6 Issue 12, December 2017

www.ijsr.net 